\title{
Numerical evaluation of a generalized Cauchy principal value
}

\author{
András Nyíri and László Baranyi
}




\title{
NUMERICAL EVALUATION OF A GENERALIZED CAUCHY PRINCIPAL VALUE
}

\author{
ANDRÁs NyíRI AND LÁSZlÓ BARANYI \\ Department of Fluid and Heat Engineering, University of Miskolc \\ 3515 Miskolc - Egyetemváros, Hungary \\ arambl@gold.uni-miskolc.hu
}

[Received July 22, 1999]

\begin{abstract}
A method is presented for the evaluation of a generalized Cauchy principal value of an improper integral. The integral is transformed into a Riemann integral which is evaluated using Romberg integration. This method is very convenient for computational purposes. The classical Cauchy principal value can be considered as a special case of the present one.
\end{abstract}

Mathematical Subject Classification: 65D30

Keywords: Cauchy principal value, improper integral, Romberg integration

\section{Introduction}

Various methods have been developed for the evaluation of principal value integrals [1-5]. Some of these are based on the Gaussian quadrature [2-4]. Methods of this type usually yield high accuracy for a given number of function evaluations. It may be difficult, however, to choose the weighing functions and the abscissas where the function is to be evaluated. Also if one desires to improve the accuracy by going to a higher order method, the calculation must be done from scratch. Kutt [5] presented a method for evaluation principal values by using finite-part integration. The authors [6] calculated a certain type of improper integrals occurring in fluid flow computations. That method has been extended and is applicable to a more general type Cauchy principal value integrals. The p.v. integral is evaluated here by the computation of a Riemann integral by using Romberg integration [7]. Although the number of function evaluations in this case is higher than that of the Gaussian quadrature, a simple and reliable method is presented by which high accuracy can be achieved within a fraction of a second CPU time even on a Personal Computer.

\section{Evaluation of generalized Cauchy principal value}

Let us consider the following improper integral

$$
I=p \cdot v \cdot \int_{x_{0}-a}^{x_{0}+a} \frac{f(x)}{h(x)-h\left(x_{0}\right)} \mathrm{d} x
$$


where

- $h(x) \neq h\left(x_{0}\right)$ for $x \neq x_{o}$ while $x \in\left[x_{0}-a, x_{0}+a\right]$,

- $a$ is a positive constant,

$-f(x)$ is continuous at $x=x_{0}$ and $f\left(x_{0}\right) \neq 0$,

- $f(x)$ is Riemann integrable over the interval $\left[x_{0}-a, x_{0}+a\right]$,

- $h(x) \in C^{1}$ in the vicinity of $x_{0}$,

- there is an interval around $x_{0}$ where the functions $f^{\prime}(x)$ and $h^{\prime \prime}(x)$ exist, therefore the following left and right side limits can be written

$$
\begin{array}{ll}
\lim _{\epsilon \rightarrow 0} f^{\prime}\left(x_{0}-\epsilon\right)=f^{\prime-}\left(x_{0}\right) & \lim _{\epsilon \rightarrow 0} f^{\prime}\left(x_{0}+\epsilon\right)=f^{\prime+}\left(x_{0}\right) \\
\lim _{\epsilon \rightarrow 0} h^{\prime \prime}\left(x_{0}-\epsilon\right)=h^{\prime \prime-}\left(x_{0}\right) & \lim _{\epsilon \rightarrow 0} h^{\prime \prime}\left(x_{0}+\epsilon\right)=h^{\prime \prime+}\left(x_{0}\right) .
\end{array}
$$

In this expression the single and double primes denote the first and second derivatives, respectively. In equation (2.1) p.v. stands for principal value.

Theorem 2.1 If the conditions above hold true, the improper integral (2.1) is equivalent to the following Riemann integral

$$
I=\int_{0}^{a} g\left(x_{0}, u\right) d u
$$

where

$$
\begin{gathered}
g\left(x_{0}, u\right)=\frac{f\left(x_{0}-u\right)}{h\left(x_{0}-u\right)-h\left(x_{0}\right)}+\frac{f\left(x_{0}+u\right)}{h\left(x_{0}+u\right)-h\left(x_{0}\right)} ; \quad \text { if } u \neq 0 \\
g\left(x_{0}, u\right)=\frac{f^{\prime-}\left(x_{0}\right)+f^{\prime+}\left(x_{0}\right)}{h^{\prime}\left(x_{0}\right)}-\frac{f\left(x_{0}\right)}{2\left[h^{\prime}\left(x_{0}\right)\right]^{2}}\left[h^{\prime \prime-}\left(x_{0}\right)+h^{\prime \prime+}\left(x_{0}\right)\right] ; \quad \text { if } u=0 .
\end{gathered}
$$

Proof Let us resolve equation (2.1) into two parts

$$
I=p . v . \int_{x_{0}-a}^{x_{0}+a} \frac{f(x)}{h(x)-h\left(x_{0}\right)} d x=\lim _{\epsilon \rightarrow 0}\left[\int_{x_{0}-a}^{x_{0}-\epsilon} \frac{f(x)}{h(x)-h\left(x_{0}\right)} d x+\int_{x_{0}+\epsilon}^{x_{0}+a} \frac{f(x)}{h(x)-h\left(x_{0}\right)} d x\right] .
$$

By introducing the transformation

$$
u=\left|x-x_{0}\right|,
$$

equation (2.6) can be reshaped to yield

$$
I=\int_{0}^{a}\left[\frac{f\left(x_{0}-u\right)}{h\left(x_{0}-u\right)-h\left(x_{0}\right)}+\frac{f\left(x_{0}+u\right)}{h\left(x_{0}+u\right)-h\left(x_{0}\right)}\right] d u \text {. }
$$

It can be seen that we arrived at equations (2.3) and (2.4) for the case when $u \neq 0$. The integrand $g\left(x_{0}, u\right)$ occurring in equation (2.7) is Riemann integrable and bounded in 
the interval $(0, a]$. When $u$ tends to zero, integrand $g\left(x_{0}, u\right)$ becomes an indeterminate of the form $0 / 0$. Now let us examine the limiting case

$$
\lim _{u \rightarrow 0} g\left(x_{0}, u\right) .
$$

By using Taylor's theorem [8], functions occurring in the integrand of equation (2.7) can be written as follows

$$
\begin{gathered}
f\left(x_{0}+u\right)=f\left(x_{0}\right)+f^{\prime}(b) u, \text { where } b \in\left(x_{0}, x_{0}+u\right) \\
f\left(x_{0}-u\right)=f\left(x_{0}\right)-f^{\prime}(c) u, \text { where } c \in\left(x_{0}-u, x_{0}\right) ; \\
h\left(x_{0}+u\right)-h\left(x_{0}\right)=h^{\prime}\left(x_{0}\right) u+h^{\prime \prime}(d) \frac{u^{2}}{2}, \text { where } d \in\left(x_{0}, x_{0}+u\right) \\
h\left(x_{0}-u\right)-h\left(x_{0}\right)=-h^{\prime}\left(x_{0}\right) u+h^{\prime \prime}(e) \frac{u^{2}}{2}, \text { where } e \in\left(x_{0}-u, x_{0}\right) .
\end{gathered}
$$

The substitution of these expressions into equation (2.4) yields

$$
g\left(x_{0}, u\right)=\frac{f\left(x_{0}\right)-f^{\prime}(c) u}{-h^{\prime}\left(x_{0}\right) u+h^{\prime \prime}(e) \frac{u^{2}}{2}}+\frac{f\left(x_{0}\right)+f^{\prime}(b) u}{h^{\prime}\left(x_{0}\right) u+h^{\prime \prime}(d) \frac{u^{2}}{2}} ; u \neq 0 .
$$

By introducing a common denominator for the fractions in equation (2.8) and applying some simple algebra, the equation can be reshaped

$$
\begin{aligned}
& g\left(x_{0}, u\right)=\frac{\left[f^{\prime}(b)+f^{\prime}(c)\right] h^{\prime}\left(x_{0}\right)-\frac{f\left(x_{0}\right)}{2}\left[h^{\prime \prime}(e)+h^{\prime \prime}(d)\right]-\left[f^{\prime}(b) h^{\prime \prime}(e)-f^{\prime}(c) h^{\prime \prime}(d)\right] \frac{u}{2}}{\left[h^{\prime}\left(x_{0}\right)\right]^{2}+h^{\prime}\left(x_{0}\right)\left[h^{\prime \prime}(d)-h^{\prime \prime}(e)\right] \frac{u}{2}-h^{\prime \prime}(e) h^{\prime \prime}(d) \frac{u^{2}}{2}} \\
& \text { if } u \neq 0 \text {. }
\end{aligned}
$$

Bearing in mind equation (2.2), expressions in equation (2.9) in the limit of $u$ tending to zero will have the following forms

$$
\begin{array}{ll}
\lim _{u \rightarrow 0} f^{\prime}(b)=f^{\prime+}\left(x_{0}\right) ; & \lim _{u \rightarrow 0} f^{\prime}(c)=f^{\prime-}\left(x_{0}\right) \\
\lim _{u \rightarrow 0} h^{\prime \prime}(d)=h^{\prime \prime}\left(x_{0}\right) ; & \lim _{u \rightarrow 0} h^{\prime \prime}(e)=h^{\prime \prime}\left(x_{0}\right) .
\end{array}
$$

Carrying out now the limit of equation (2.9) when $u$ tends to zero gives equation (2.5) to be proved

$$
\lim _{u \rightarrow 0} g\left(x_{0}, u\right)=\frac{f^{\prime-}\left(x_{0}\right)+f^{\prime+}\left(x_{0}\right)}{h^{\prime}\left(x_{0}\right)}-\frac{f\left(x_{0}\right)}{2\left[h^{\prime}\left(x_{0}\right)\right]^{2}}\left[h^{\prime \prime-}\left(x_{0}\right)+h^{\prime \prime+}\left(x_{0}\right)\right] .
$$

With this theorem 2.1 is proved.

Remark 2.1 By applying the theorem proved above the evaluation of the generalized Cauchy principal value can be reduced to that of a Riemann integral by using various types of numerical quadrature. The advantage of the method lies in its simplicity. Computational experience gained shows that there is no need to evaluate the integrand at the close proximity of the pole. In this way the error due to the summing up of two large numbers of different signs can be avoided. 
Remark 2.2 In a case when the derivatives $f^{\prime}(x)$ and $h^{\prime \prime}(x)$ are continuous at $x=x_{0}$, equation (2.5) will have the following form

$$
g\left(x_{0}, u\right)=2 \frac{f^{\prime}\left(x_{0}\right)}{h^{\prime}\left(x_{0}\right)}-\frac{f\left(x_{0}\right)}{\left[h^{\prime}\left(x_{0}\right)\right]^{2}} h^{\prime \prime}\left(x_{0}\right) ; \quad \text { if } u=0 .
$$

Remark 2.3 The classical Cauchy principal value is obtained by substituting

$$
h(x) \equiv x
$$

into equation (2.1). In this case equations (2.4) and (2.5) are simplified to the following equations

$$
\begin{gathered}
g\left(x_{0}, u\right)=\frac{f\left(x_{0}+u\right)-f\left(x_{0}-u\right)}{u}, \quad \text { if } u \neq 0 \\
g\left(x_{0}, u\right)=f^{\prime-}\left(x_{0}\right)+f^{\prime+}\left(x_{0}\right), \quad \text { if } u=0 .
\end{gathered}
$$

Remark 2.4 The method shown in [6] worked out for the computation of incompressible inviscid flow around airfoils can also be considered as a special case of the present method. It can easily be seen that the evaluation of the integral in [6]

$$
I=p \cdot v \cdot \int_{x_{0}-a}^{x_{0}+a} \frac{f(x)}{\cos x-\cos x_{0}} \mathrm{~d} x
$$

can be carried out by the method shown here. The substitution of

$$
h(x) \equiv \cos x
$$

into equation (2.1) leads to equation (2.10). Now it is straightforward that the integrands occurring in the two cases coincide

$$
\begin{aligned}
& g\left(x_{0}, u\right)=\frac{f\left(x_{0}-u\right)}{\cos \left(x_{0}-u\right)-\cos x_{0}}+\frac{f\left(x_{0}+u\right)}{\cos \left(x_{0}+u\right)-\cos x_{0}}, u \neq 0 \\
& g\left(x_{0}, u\right)=-\frac{f^{\prime-}\left(x_{0}\right)+f^{\prime+}\left(x_{0}\right)}{\sin x_{0}}+f\left(x_{0}\right) \frac{\cos x_{0}}{\sin ^{2} x_{0}}, \quad u=0, \sin x_{0} \neq 0 .
\end{aligned}
$$

\section{Examples}

The computations of the following three examples were performed in double precision on an IBM PC. The evaluation of the integral (2.3) was carried out by dividing the domain of integration into $n$ equidistant part-intervals and applying the Romberg integration. Calculations were repeated for several different values of $n$; hence, its effect on the convergence of the result can also be studied (see Tables 3.1-3.3).

(a)

$$
I=p . v . \int_{0.5}^{1.5} \frac{\mathrm{d} x}{x^{3}-1}
$$


Comparing equations (2.1) and (3.1) it can easily be seen that in this case

$$
f(x) \equiv 1 ; h(x)=x^{3} ; a=0.5 .
$$

The primitive function $F(x)$ of the integrand in (3.1) is also available in analytic form $[9]$

$$
F(x)=\frac{1}{6} \log \left[\frac{(x-1)^{2}}{\left|x^{2}+x+1\right|}\right]+\frac{1}{\sqrt{3}} \tan ^{-1}\left(\frac{-2 x-1}{\sqrt{3}}\right) .
$$

Hence integral (3.1) can be evaluated as

$$
I=p . v . \int_{0.5}^{1.5} \frac{d x}{x^{3}-1}=\lim _{\epsilon \rightarrow 0}\left\{[F(x)]_{0.5}^{1-\epsilon}+[F(x)]_{1+\epsilon}^{1.5}\right\}=-0.342563258354480 .
$$

This accurate result can be compared to that of the one obtained from the present numerical approximation. The computational results for different values of the partintervals $n$ are shown in Table 3.1

Table 3.1

\begin{tabular}{|c|c|}
\hline $\mathrm{n}$ & $\mathrm{I}$ \\
\hline 4 & -0.342563143279927 \\
\hline 8 & -0.342563258270464 \\
\hline 16 & -0.342562258354677 \\
\hline 32 & -0.342563258354481 \\
\hline 64 & -0.342563258354480 \\
\hline
\end{tabular}

(b)

$$
I=p \cdot v \cdot \int_{-1}^{1} \frac{e^{x}}{x} d x
$$

Although as far as our knowledge goes, the primitive function does not exist in this case, Table 3.2 displays a rapid convergence.

Table 3.2

\begin{tabular}{|c|c|}
\hline $\mathrm{n}$ & $\mathrm{I}$ \\
\hline 4 & 2.11450191410513 \\
\hline 8 & 2.11450175080131 \\
\hline 16 & 2.11450175075146 \\
\hline 32 & 2.11450175075146 \\
\hline 64 & 2.11450175075146 \\
\hline
\end{tabular}

(c)

$$
I=p \cdot v \cdot \int_{0.5}^{1.5} \frac{x^{2}}{x^{4}-1} \mathrm{~d} x
$$


The primitive function $F(x)$ of the integrand in the equation (3.2) is also available in analytical form [9]

$$
F(x)=\frac{1}{4}\left[\log \left|\frac{x-1}{x+1}\right|+2 \tan ^{-1} x\right] .
$$

Hence integral (3.2) can be evaluated as

$$
I=p . v . \int_{0.5}^{1.5} \frac{x^{2}}{x^{4}-1} \mathrm{~d} x=\lim _{\epsilon \rightarrow 0}\left\{[F(x)]_{0.5}^{1-\epsilon}+[F(x)]_{1+\epsilon}^{1.5}\right\}=0.131866651181764
$$

This accurate result shows good agreement with the results of our computations belonging to different values of $n$, contained in Table 3.3

Table 3.3

\begin{tabular}{|c|c|}
\hline $\mathrm{n}$ & $\mathrm{I}$ \\
\hline 4 & 0.131866662407660 \\
\hline 8 & 0.131866651984160 \\
\hline 16 & 0.131866651180677 \\
\hline 32 & 0.131866651181764 \\
\hline 64 & 0.131866651181764 \\
\hline
\end{tabular}

The fast convergence of the results can be observed in Tables 3.1-3.3. The advantages of the method are its simplicity, high accuracy and reliability. Although there may be faster methods, the actual CPU times for the evaluation of the sample cases above were small fractions of a second even on a PC.

\section{REFERENCES}

[1] Mangler, K.W.: Improper Integrals in Theoretical Aerodynamics, Report No. Aero 2424, Royal Aircraft Establishment, Farnborough, 1951.

[2] Brebila, C.A., Telles, J.C.F. and Wrobel, L.C.: Boundary Element Techniques, Springer Verlag, Berlin, 1984.

[3] Paget, D.F. and Eliot, D.: An algorithm for the numerical evaluation of certain Cauchy principal value integrals, Numer. Math., 19, (1972), 373-385.

[4] Hunter, D.B.: Some Gauss-type formulae for the evaluation of Cauchy principal values of integrals, Numer. Math., 19, (1972), 419-424.

[5] KutT, H.R.: The numerical evaluation of principal value integrals by finite-part integration, Numer. Math., 24, (1975), 204-210.

[6] Nyíri, A., Baranyi, L. and Kalmár, L.: A numerical method to solve an integral equation of singular kernel, Micro-CAD '94 International Computer Science Meeting, Miskolc, Hungary, Section L, (1994), 28-35.

[7] Ferziger, J.H.: Numerical Methods for Engineering Application, John Wiley \& Sons, New-York, 1981. 
[8] Ross, K.A.: Elementary Analysis, The Theory of Calculus, Springer Verlag, New-York, 1980.

[9] Moriguchi, S., Udagawa, K. and Hitotsumatsu, S.: Mathematical Formulae I, Differentiation, Integration, Curved Surfaces, (in Japanese), Iwanami Publisher, Tokyo, 1975. 